\title{
LEE: A Photorealistic Virtual Environment for Assessing Driver-Vehicle Interactions in Self-driving Mode
}

\author{
Saad Minhas ${ }^{1}$, Aura Hernández-Sabaté ${ }^{2(凶)}$, Shoaib Ehsan ${ }^{1}$, \\ Katerine Díaz-Chito ${ }^{2}$, Ales Leonardis ${ }^{3}$, Antonio M. López ${ }^{2}$, \\ and Klaus D. McDonald-Maier ${ }^{1}$ \\ 1 University of Essex, Colchester, UK \\ 2 Computer Vision Center and Universitat Autónoma de Barcelona, \\ Barcelona, Spain \\ aura@cvc.uab.cat \\ 3 University of Birmingham, Birmingham, UK
}

\begin{abstract}
Photorealistic virtual environments are crucial for developing and testing automated driving systems in a safe way during trials. As commercially available simulators are expensive and bulky, this paper presents a low-cost, extendable, and easy-to-use (LEE) virtual environment with the aim to highlight its utility for level 3 driving automation. In particular, an experiment is performed using the presented simulator to explore the influence of different variables regarding control transfer of the car after the system was driving autonomously in a highway scenario. The results show that the speed of the car at the time when the system needs to transfer the control to the human driver is critical.
\end{abstract}

Keywords: Simulation environment $\cdot$ Automated driving · Drivervehicle interaction

\section{Introduction}

The last few decades have seen a dramatic increase in the number of vehicles utilizing Advanced Driver Assistance Systems (ADAS), such as intelligent headlights [3], lane change assistance [2], and even the first attempts of automatic driving systems $[6,14,15,18]$. Although currently far from having feasible totally automated driving systems, there are several intermediate levels of driving automation for on-road vehicles, according to the SAE international standard J3016 [12], based on the system core functionality. Its level 3 specifies that the automated driving system performs all aspects of dynamic driving task with the expectation that the driver will recover the car's control when required. Thus, the human driver can perform other activities while the system is driving autonomously. This gives rise to an important question: At which moment and how can the automated driving system return the control to the driver? 
The answer to this question depends on several aspects, such as the activity of the driver, his/her general state and possible reaction, the particular state of the environment and the current action of the car. All these aspects should be carefully analyzed without compromising road security, and hence require a simulated environment for research, development and testing purposes.

This paper presents a low-cost, extendable and easy-to-use (LEE) simulation environment which allows to explore autonomous driving research. To demonstrate its utility, an experiment is performed using this simulator by designing two scenarios where the driver is alerted by a visual and acoustic alarm and asked to take over the control. Regardless of the activity of the driver, perception reaction time (PRT) has to be taken into account before making any decision [10], among other variables. Moreover, the reaction times pertaining to arm and feet are different [11]. Several variables are examined in this paper, such as the speed of the car at that moment, the reaction time of the arms (needed to reach the steering wheel) and the reaction time of the feet (needed to reach the brake pedal). The results show that the speed of the car at the time of triggering the alarm is critical. It can be observed that PRT of arms are greater than PRT of feet, which should be taken into account in further analysis. Figure 1(a) shows the driver simulator hardware setup while Fig. 1(b) provides a screenshot of the simulation recording with all the variables taken into account.

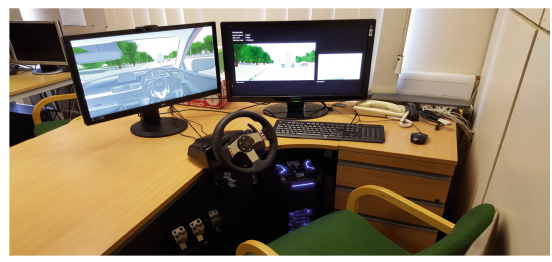

(a)

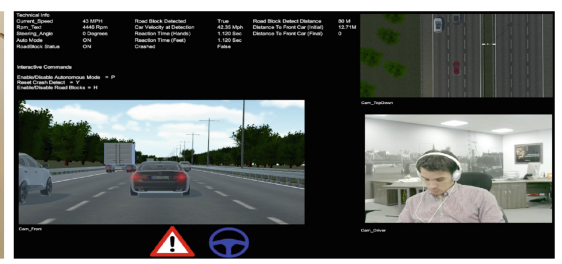

(b)

Fig. 1. Simulator hardware (a) and an example of the simulation recording (b).

\section{Related Work}

Simulation Environments. The first interactive driving simulator was introduced in 1960 [5]. TRAFFIS is an industrial grade driver simulator relying on a reconfigurable approach [1]. Similarly, $3 \mathrm{xD}$ is a driver-in-loop multi-axis driving simulator. The VTI's simulator consists of a partial car mock-up, hydraulic movement and a moving screen [9]. These simulators are high-cost and bulky requiring specialist dedicated hardware, with a Toyota's simulator costing in the region of 30 million pound sterling [17].

Perception Reaction Time. PRT of human drivers is an active research area within the driving performance domain, where it plays an important role in road incidents $[4,7,8]$. Green [4] highlights that the most important variable is 
driver's expectation. Jurecki et al. [7] confirm that reaction time is approximately a linear function of Time To Collision (TTC). Svetina [13] concludes that mean reaction time and inter-individual variability progressively increases with age. It is worth mentioning here that all these studies are carried out on active users while actually driving a vehicle.

\section{Simulation Environment}

This section presents the simulation environment 'LEE'. For building an efficient and inexpensive simulator, a custom workstation equipped with an Intel i7 processor, an NVIDIA GTX Titan Graphics card with 12GB of usable VRAM, two HD monitors, a HD Webcam and a Logitech G27 Wheel and pedal Set are used. On the software side, Autodesk 3DS Max is used to model and develop virtual assets for the driver simulator. This includes the driver's car, other traffic cars as well as road surfaces. Adobe Photoshop is used in the creation of $2 \mathrm{D}$ elements, which includes detailing on the modelled cars as well as the road sections. Finally, Unity3D is used to tackle the interactive challenges of the simulator.
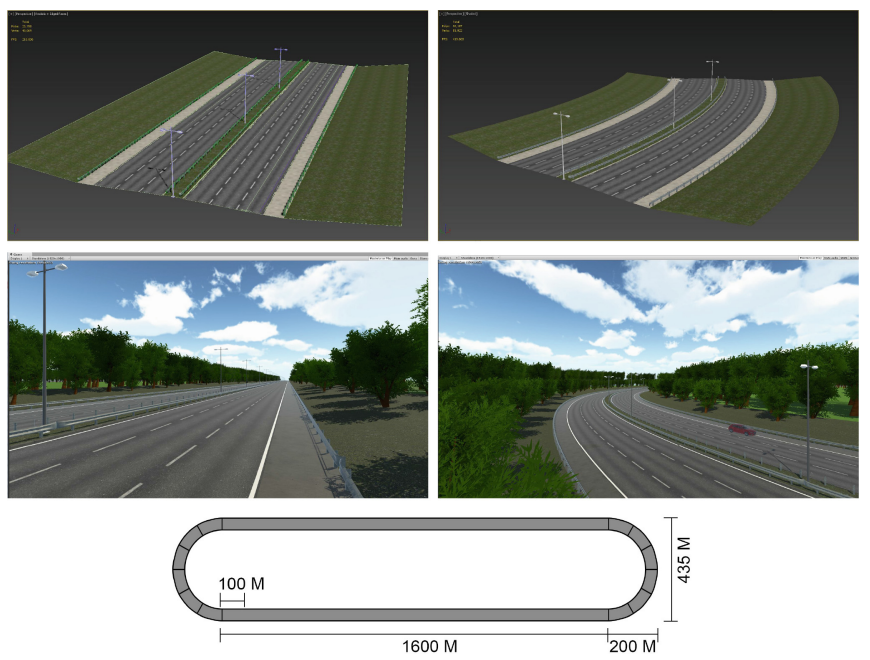

Fig. 2. Road surface. Road sections (top row), Unity3D road environment (middle row) and road model dimensions (bottom row).

Major 3D assets that are created involve the vehicular models and the environment assets. The vehicle models provided by the Mission Group [16] and the traffic cars are then processed to optimize them for real-time applications. This includes stripping down any unwanted details on the models. In particular, the interiors of these vehicles are reduced quite substantially as they are never seen in the actual application. A Mazda 3 has been chosen as the model. The driver car's interior is also optimized to resemble the real life counterpart as close as possible. 
The road surface is modelled in 3 dsmax by using reference imagery from the internet. It is loosely based on a three lane section of M25 motorway around London. In order to keep the modular aspect of the environment intact, only two road sections are produced, a straight road section consisting of 100 meters, and an angular portion of 25 degrees. These two road sections help in creating different looking road environments within Unity3D. Basic trees are populated on either side of the road and a suitable sky environment is added to further enhance the realism of the virtual environment. The finished 3D assets including the vehicles and the road sections are then imported into Unity3D. The two road sections are cloned into multiple copies and are put together to form a looping M25 environment, which is approximately 4 miles long and is used as a base for the virtual world experiments. Figure 2 shows the road surface, including the two road sections at the top, the complete environment within Unity3D in the middle and the complete road model dimensions at the bottom.

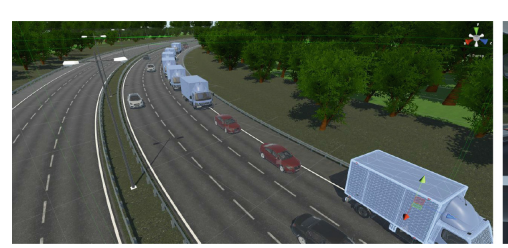

(a)

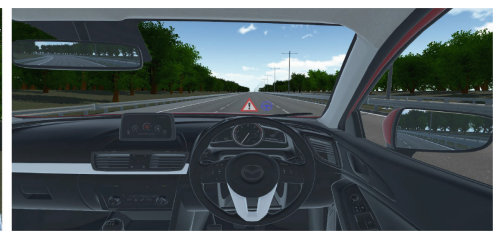

(b)

Fig. 3. Virtual world. (a) Traffic simulation and (b) Main car interior rig

The traffic cars are populated by using a third party plugin called ITS (Intelligent Traffic System). Cars can be seen maneuvering throughout the virtual environment, overtaking other traffic vehicles and maintaining their specific lanes. The system is rigged in such a manner that the left most lane would have a speed of no more than $55 \mathrm{mph}$ and will have a greater number of lorries, whereas the middle lane would have a $60 \mathrm{mph}$ limit with medium and small sized cars. Finally, the right most lane has a speed limit of $70 \mathrm{mph}$. The cars that are populated do not necessarily keep a constant speed and it varies from car to car for producing a more realistic traffic simulation. The cars also stop when they detect a blockage in front of the road. The simulations are random every time the application is executed, hence giving a realistic and unperceived situation with every new session. Figure 3(a) shows the traffic system in action.

The main car model requires the most amount of rigging, as this needs to be an area where the driver would be interacting the most. Main components of the interior of the car are rigged in a realistic manner (Fig. 3(b)). This includes rigging the steering wheel, RPM needle, pedals and a fully functional Adaptive Cruise Control System. Moreover, the general car physics rig is based on the standard Unity3D Car Controller model. This gives a definitive advantage over the future upgrade of the physics model. The rear view mirrors reflect a virtual camera that is projected on the surface of the mirrors via Render to Texture 
approach. Finally, the main car has a fully functional Autonomous Driver mode which has the capacity of maintaining a particular distance from the car in front by using the primary proximity zone. It can also brake hard when the car in front enters the secondary proximity zone. Figure 4 shows how the Adaptive Distance Awareness works in the driver simulator.

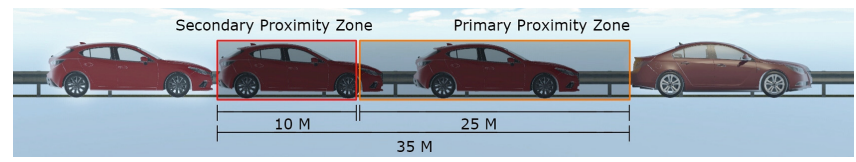

Fig. 4. Autonomous proximity sensor model

\section{Application}

Experimental Setup. While the car is running in autonomous mode, two different scenarios are defined in this experiment using LEE: (1) the subject is not looking at the road, but attentive with hands on the wheel, which serves as a baseline so that other scenarios can be used to compare the results for evaluation; (2) the subject is on the phone checking social media. In both cases, once the car detects a road block at a random distance ahead, it triggers an alarm, at which point the subject has to take back control in order to avoid a crash. LEE records the video of the subject, and several variables involved in the process, such as Hands/Feet PRT and the speed at which the alarm was triggered.

A total of 10 subjects aged between 26 and 62 years were involved in the experiment. Each trial contains 12 sessions, 6 for each scenario. The distance at which the road block is detected is set to 60 meters in 3 sessions and 80 meters in the other 3. We have compared the hands and feet PRT by means of the computation of their ranges (mean \pm std) and have also explored the influence of some of the variables recorded such as Hands/Feet PRT and speed.

Results and Analysis. The subjects were found to keep the wheel in a static position unchanged from the Autonomous mode was in, thus in this context, the driver appears to concentrate on control of the pedals first. This result is also evident when we compare the Hands/Feet PRT of both the scenarios, in which Hands PRT are significantly greater than Feet PRT. Table 1 summarizes the ranges for Hands/Feet PRT in both scenarios.

Figure 5 shows the influence of three variables, Hands/Feet PRT and speed, where blue and red colors show crash/no crash results, respectively. Dots and

Table 1. Comparison of Hands/Feet PRT in both scenarios

\begin{tabular}{l|l|l|l}
\hline & Hands & Feet & p-value \\
\hline 1st scenario & $1.67 \pm 1.61$ & $1.26 \pm 0.45$ & 0.0034 \\
\hline 2nd scenario & $2.71 \pm 1.91$ & $1.42 \pm 0.34$ & $8.41 \times 10^{-8}$ \\
\hline
\end{tabular}


circles represent results for scenario 1 and scenario 2, respectively. It can be observed that the speed of the car when the alarm was triggered is a determinant variable in both scenarios since we can appreciate two separate clusters in the speed direction. As opposed to this, two separate clusters in the direction of feet or hands PRT cannot be seen.
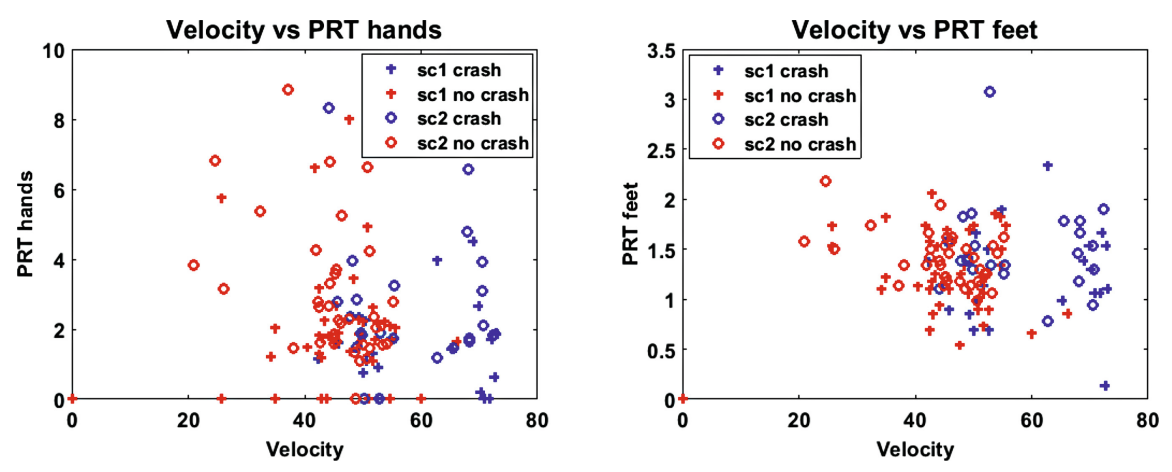

Fig. 5. Speed against PRT in both scenarios (Color figure online)

\section{Conclusion and Future Directions}

This paper has presented an affordable, customizable and easy-to-use simulation environment for facilitating research on driver-vehicle interaction for level 3 autonomous driving. The simulator is designed to be deployed at any location with the minimum amount of hardware peripherals, which significantly reduces the cost of the entire setup.

The results of an experiment at small scale, performed to highlight the utility of LEE, show that the speed of the car at the transfer moment is critical for ensuring vehicle safety. Distance to the front car is not a $100 \%$ plausible variable, because there are situations where a driver would steer clear of any cars, or the initial distance before the road block between the front car and the user car is quite large. In future, we plan to increase the number of scenarios as the cognitive load of the driver is another variable that should be taken into account.

Besides, there are more potential uses of LEE to improve the perception engines of self-driving cars, such as driver state recognition and PRT prediction in function of both external (road, traffic, environment, weather, lighting...) and internal (driver, passengers, car configuration...) situations. As well, another question arises, how to maintain and check the driver attentiveness during selfdriving? Computer vision and Artificial Intelligence will play a crucial role in the particular case of monitoring the driver and recognizing her/his attentiveness and general state and deciding how to transfer the car's control. LEE will allow to do all the tests in a road safety manner, which will be approached further.

Acknowledgements. This work is supported by the Spanish MICINN project TRA2014-57088-C2-1, by the Secretaria d'Universitats i Recerca del Departament d'Economia i Coneixement de la Generalitat de Catalunya (2014-SGR-1506). 


\section{References}

1. Abdelgawad, K., Abdelkarim, M., Hassan, B., Grafe, M., Gräßler, I.: A modular architecture of a PC-based driving simulator for advanced driver assistance systems development. In: 2014 15th International Workshop on Research and Education in Mechatronics (REM), pp. 1-8. IEEE (2014)

2. Bartels, A., Meinecke, M.M., Steinmeyer, S.: Lane change assistance. In: Eskandarian, A. (ed.) Handbook of Intelligent Vehicles, pp. 729-757. Springer, London (2012)

3. Eum, S., Jung, H.G.: Enhancing light blob detection for intelligent headlight control using lane detection. IEEE Trans. Intell. Transp. Syst. 14(2), 1003-1011 (2013)

4. Green, M.: "How long does it take to stop?" methodological analysis of driver perception-brake times. Transp. Hum. Factors 2(3), 195-216 (2000)

5. Hulbert, S., Wojcik, C.: Driving simulator research. Highw. Res. Board Bull. 261, $1-13(1960)$

6. Jitsukata, E., Kobayashi, S., Tamura, K.: Automatic driving system. US Patent 6,169,940, 2 January 2001

7. Jurecki, R.S., Stańczyk, T.L.: Driver reaction time to lateral entering pedestrian in a simulated crash traffic situation. Transp. Res. Part F: Traffic Psychol. Behav. 27, 22-36 (2014)

8. Jurecki, R.S., Stańczyk, T.L., Jaśkiewicz, M.J.: Driver's reaction time in a simulated, complex road incident. Transport 1-11 (2014). http://dx.doi.org/ 10.3846/16484142.2014.913535

9. Knutsson, U.K.: Swedish national road and transport research institute. IATSS Res. 27(2), 88-91 (2003)

10. Kuang, Y., Qu, X., Weng, J., Etemad-Shahidi, A.: How does the driver's perception reaction time affect the performances of crash surrogate measures? PLoS One 10(9), e0138617 (2015)

11. Martin, S., Ohn-Bar, E., Tawari, A., Trivedi, M.M.: Understanding head and hand activities and coordination in naturalistic driving videos. In: 2014 IEEE Intelligent Vehicles Symposium Proceedings, pp. 884-889. IEEE (2014)

12. SAE levels driving automation: http://cyberlaw.stanford.edu/blog/2013/12/ sae-levels-driving-automation. Accessed 29 July 2016

13. Svetina, M.: The reaction times of drivers aged 20 to 80 during a divided attention driving. Traffic Inj. Prev. (2016, in press)

14. Tesla driver dies in first fatal crash while using autopilot mode. https://www. theguardian.com/technology/2016/jun/30/tesla-autopilot-death-self-driving-carelon-musk. Accessed 29 July 2016

15. Tesla says autopilot involved in second car crash. http://www.bbc.com/news/ technology-36783345. Accessed 29 July 2016

16. The Mission. http://www.themission.co.uk/news/bigdog-goes-virtual-for-mazda2launch/. Accessed 29 July 2016

17. Toyota's Driving Simulator Review. http://www.telegraph.co.uk/motoring/ roadsafety/6598418/Toyotas-30-million-drivingsimulator-review.html.

Accessed 29 July 2016

18. Zhang, W., Mei, T., Liang, H., Li, B., Huang, J., Xu, Z., Ding, Y., Liu, W.: Research and development of automatic driving system for intelligent vehicles. In: Sun, F., $\mathrm{Hu}$, D., Liu, H. (eds.) Foundations and Practical Applications of Cognitive Systems and Information Processing. AISC, vol. 215, pp. 675-684. Springer, Heidelberg (2014). doi:10.1007/978-3-642-37835-5_58 